Isabell Neise

\title{
Vergleichende Gesprächsforschung \\ Bericht über die 21. Arbeitstagung zur Gesprächsforschung vom 21.-23. März 2018 am Institut für Deutsche Sprache (Mannheim)
}

Die 21. Arbeitstagung zur Gesprächsforschung mit dem Rahmenthema „Vergleichende Gesprächsforschung“ fand vom 21.-23. März 2018 am Institut für Deutsche Sprache in Mannheim statt. Das Ziel der Tagung war es, Forscherinnen und Forscher zusammenzubringen, die authentische Interaktionsdaten aus vergleichender Perspektive untersuchen. Das Rahmenthema der Tagung ergab sich aus dem steigenden Interesse an vergleichenden Fragestellungen innerhalb konversations- und gesprächsanalytischer Untersuchungen. Die Tagung nahm gezielt Vorgehensweisen und Methoden bei der Durchführung vergleichender Untersuchungen in den Blick. Die Vorträge, ${ }^{1}$ Projektpräsentationen und Datensitzungen erörterten 1. das Vergleichen als analytische Grundoperation der Konversationsund Gesprächsanalyse, 2. Vergleiche alternativer Ressourcen und Praktiken für spezifische Handlungen und Aktivitäten in der Interaktion sowie 3. methodologische Herausforderungen einer vergleichenden Gesprächsforschung.

\section{Vorträge - Tag 1}

Eröffnet wurde die Tagung durch den Direktor des IDS, Ludwig M. Eichinger (10/200207/2018). Nach der Begrüßung der Gäste folgte die Verleihung des mit 1000 Euro dotierten Dissertationsförderpreises des Vereins für Gesprächsforschung e. V. durch Cordula Schwarze an Clarissa Weiß (Freiburg) für ihr Dissertationsprojekt „Blick und Turntaking in Face-to-face Interaktionen. Multimodale Interaktionsanalysen triadischer Gesprächssituationen mit Hilfe von Eye-Tracking“, das den Zusammenhang von Blickverhalten und Turntaking untersucht.

Nach der Begrüßung und der Verleihung des Dissertationspreises folgte der Eröffnungsvortrag von Jörg Zinken und Silke Reineke (Mannheim), die in den Gegenstand der vergleichenden Gesprächsforschung als gängiges methodisches Vorgehen innerhalb traditioneller Sozialwissenschaften, der Konversationsanalyse sowie Gesprächsforschung einführten. Die Darstellung wurde in Fragen, Wege und Lösungsansätze hinsichtlich der eingereichten Abstracts unterteilt. Hervorgehoben wurde insbesondere die Rolle der Vergleichsgrundlage, als stetes, gleichbleibendes Moment jeder auf den Vergleich zugeschnittenen Untersuchung.

\footnotetext{
Aufgrund des Überblickscharakters dieses Berichtes können nicht alle Beiträge eingehend besprochen werden. Eine ausführlichere Version dieses Berichts ist jedoch in „Gesprächsforschung Online“erschienen (vgl. Neise 2018, In: Sprachreport 34, 3, S. 52-55, Online unter: pub.ids-mannheim.de/laufend/ sprachreport/pdf/sr18-3.pdf, Stand: 18.9.2018). Weitere Literaturhinweise und die vollständigen Abstracts der erwähnten Beiträge sind zudem dauerhaft abrufbar unter www.gespraechsforschungonline.de/2018.html (Stand: 21.2.2019).
} 
Der Vortrag von Lorenza Mondada (Basel) ,,,mimole e hä i gärn ka‘: A multimodal comparative analysis of requests" betrachtete Nachfragehandlungen nach Käse aus einer multimodalen und sprachvergleichenden Perspektive. Das der Untersuchung zugrundeliegende Videokorpus besteht aus Käse-Verkaufsgesprächen in Käsefachgeschäften und auf Märkten, die in 15 europäischen Städten und elf verschiedenen Sprachen aufgezeichnet wurden. Sie zeigte, dass Bestellungen der Kaufenden mit verschiedenen Handlungsformaten durchgeführt werden -1 . durch eine Nennung des Käsenamens, 2. Zeigegesten (auch in Verbindung mit der Namensnennung oder als indexikalische Gesten), 3. Beschreibungen des Käses (generisch vs. spezifisch) oder 4. Fragen. Des Weiteren konnte sie herausstellen, dass die Nachfragehandlung, die Art und Weise, wie Käse beschrieben wird und der Verlauf der Verkaufsgespräche von der Käseaffinität einer jeweiligen Kultur abhängig zu sein scheinen.

Mike Huiskes (Groningen) beschäftigte sich in seinem Vortrag „Learning to guide surgical teams: A comparative study of methods of recruitment used by residents and surgeons in the OR" mit Operationen als situierter und kollaborativer Praxis. Seine Analysen zeigten Unterschiede in den sprachlichen und körperlichen Praktiken, die Mitglieder eines Operationsteams für die Organisation ihrer Zusammenarbeit einsetzen. Die Untersuchung zeigte, dass Assistenzärztinnen und -ärzte recruitments fast durchgehend sprachlich produzieren und eine größere Vielfalt an Praktiken für die Handlungskoordination mit dem Team nutzen als Oberärztinnen und -ärzte. Im Gegensatz zu den Assistenzärztinnen und -ärzten, die recruitments vermehrt sprachlich produzierten, verwendete das Operationsteam vermehrt körperliche recruitments.

Im Vortrag „Gesprächseröffnungen in helfenden Professionen - Ein Vergleich von Erstgesprächen in der sozialen Arbeit und der Psychotherapie“ zeigten Cornelia Rüegger und Claudio Scarvaglieri (Olten/Basel), dass in therapeutischen Erstgesprächen die Rolle der/des Therapeutin/Therapeuten deutlich passiver ist, als die der/des Sozialarbeiterin/ Sozialarbeiters. Zurückzuführen sei dies auf die verschiedenen institutionellen Ausgangspositionen - während in der Sozialen Arbeit durch die sozialstaatliche Anbindung der Hilfeauftrag extern in der Form eines Kontrollauftrags vergeben wird, wird in der Psychotherapie der Hilfeauftrag freiwillig durch die/den Patientin/Patienten vergeben.

Marc Alexander und Elisabeth Stokoe (Loughborough) zeigten in ihrem Vortrag „Neighbour problems: Formulating noise complaints across different dispute resolution organization“, dass Mieter/innen unterschiedliche Strategien dafür nutzen, eine Beschwerde über Lärmbelästigung durch eine/einen Nachbarin/Nachbarn vorzutragen, je nachdem ob der Anruf bei „,mediation call services“ oder „environmental health services“ vorgetragen wurde. Bei Telefonaten mit „mediation call services“ wurde die/der Nachbarin/ Nachbar zum Beispiel unmittelbar als Agens der Ruhestörung genannt, bei ,environmental health services“ wurde das Agens wiederum ausgelassen („It's about my neighbour“ vs. „I need to speak to someone about disturbance“).

Der Vortrag „Opening business-to-business prospecting 'cold' calls“ von Bogdana Huma, Elizabeth Stokoe und Rein Ove Sikveland (Loughborough) verglich Strategien, die in „Business-to-Business cold calls“ dafür genutzt werden, mit einer/einem Verantwortlichen für ein Kundengespräch verbunden zu werden. Je nachdem, ob bereits ein Kontakt zu einem Verantwortlichen eines Unternehmens bestand oder nicht (,lukewarm calls“ vs. „freezing cold calls") unterscheiden sich die angewandten sprachlichen Strategien - Erstere sind personalisierter, indem namentlich nach dem Erstkontakt gefragt wird und auf 
das vorherige Gespräch verwiesen wird. Im Gegensatz dazu verfolgen Letztere das Ziel, überhaupt erst mit einer/einem Verantwortlichen verbunden zu werden.

\section{Projektpräsentationen}

Am Nachmittag fanden zudem die Projektpräsentationen statt, die aus 15-minütigen Vorträgen mit anschließender Diskussion bestehen und laufende oder geplante Projekte aus dem Bereich der Gesprächsforschung vorstellen. Die Präsentationen sind nicht an das Rahmenthema der Tagung gebunden:

- David Suderland stellte erste Ergebnisse seiner Dissertation „Praktiken des Vorlesens und Zeigens - ein Vergleich mediatisierter und nicht-mediatisierter Bewertungssequenzen in Face-to-Face Interaktionen" vor.

- Katja Arens präsentierte in ihrem Vortrag,,Strukturieren und Evaluieren im Gespräch. Lexikalische Partikeln als Ressource der Interaktionssituation“ erste Ergebnisse ihrer Dissertation am Beispiel der Partikel gut.

- Sarah Torres Cajo sprach über „Positionierungsaktivitäten in der Interaktion“ und zeigte, wie ihr Dissertationsprojekt versucht, sprachliche Praktiken der Positionierung aufzudecken.

- Isabell Neise führte in ihr Dissertationprojekt „Involvementstrategien im sozialen Netzwerk Twitch" ein und verdeutlichte aus den Affordanzen des Videoportals resultierende Beteiligungs- und Interaktionsmöglichkeiten.

Im Anschluss an die Vorträge des ersten Tagungstages fand die Mitgliederversammlung des „Vereins für Gesprächsforschung e. V.“ statt.

\section{Vorträge - Tag 2}

Den zweiten Tagungstag eröffnete Mark Dingemanse (Nijmegen) mit seinem Vortrag „Advances in the typology of conversational structures“. Zunächst führte er in Typologien ein, die systematische Vergleiche zwischen sprachlichen Strukturen ermöglichen; er zeigte dann auf, dass insbesondere Typologien konversationeller Strukturen rar sind, obwohl sie sich in besonderem Maß dafür eignen, Sprache im Kontext von Kognition, Kultur und Kommunikation zu untersuchen. Er verwies darauf, dass gerade anhand informeller Faceto-Face Interaktionen die Ökologie einer Sprache aufgezeigt werden kann, da diese die Hauptorte des Lernens und der Sozialisation sind, was Sprache im Kontext von Kognition, Kultur und Kommunikation untersuchbar macht. Am Beispiel fremd-initiierter Reparaturen mittels huh und multimodaler recruitments konnte er zeigen, dass es kultur- und sprachübergreifende, allgegenwärtige Praktiken zur Erreichung kommunikativer Ziele gibt.

Emma Betz, Henrike Helmer und Arnulf Deppermann fokussierten in ihrem Vortrag „OKAY: Eine sprachübergreifende, komparative Studie eines Internationalismus“ den Gebrauch der Partikel OKAY und stellten erste Ergebnisse eines komparativen Projektes vor, das zum Ziel hat, das Verwendungsspektrum von $O K A Y$ in 14 unterschiedlichen Sprachen aufzuzeigen und von anderen Diskurspartikeln abzugrenzen. Vergleiche syntaktisch nicht integrierter $O K A Y \mathrm{~s}$ innerhalb von Turns, alleinstehender $O K A Y \mathrm{~s}, O K A Y \mathrm{~s}$ in interaktionsorganisatorischen Verwendungen sowie in generellen sequenziellen Verwendungen in ausgewählten Sprachen, spezifischen Handlungsaktivitäten und Settings zeigten, dass $O K A Y$ von Sprecher/innen für das Anzeigen von Verstehen, aber auch Erwartungsdiskrepanzen 
verwendet wird und die Prosodie bei der Bestimmung der Funktion der Partikel eine entscheidende Rolle spielt.

Der Vortrag von Jörg Zinken, Lorenza Mondada, Giovanni Rossi, Marja-Leena Sorjonen und Matylda Weidner (Mannheim/Basel/Helsinki/Bydgoszcz) „PECII: Ein Parallelkorpus für die vergleichende Gesprächsforschung" behandelte die Anforderungen an den Aufbau sprachvergleichender Videokorpora informeller Interaktionen, deren gemeinsamer Referenzpunkt Handlungen und Praktiken der Interaktionsteilnehmer/innen in konkreten Aktivitäten sind. Um sprachliche Praktiken und Aktivitäten international vergleichbar zu machen, müssen beispielsweise die Aufnahme-Settings gleich aufgebaut werden (z. B. vier Personen sitzen an einem Tisch und spielen ein Brettspiel).

Giorgio Antonioli und Manuela Moroni (Trento) zeigten in ihrem Vortrag „Der Bedeutungsanteil der Intonation an der Ausführung von Frageaktivitäten im Italienischen und Deutschen", dass informationssuchende und raumdeiktische Fragen in deutschen und italienischen Alltagsgesprächen, in denselben Kontexten und für gleiche kommunikative Aufgaben, eine ähnliche intonatorische Gestalt aufweisen. Zusammenfassend räsonierten sie, dass nicht die Syntax die intonatorische Gestaltung der Fragen bestimmt, sondern die mit der Frage auszuführende Aktivität.

Minka Džanko (Sarajevo) präsentierte in ihrem Vortrag „Pronominale Anredeformen in Arzt-Patient-Gesprächen. Ein Vergleich im Deutschen und Bosnischen“, dass bosnische Arzt-Patienten-Gespräche informeller ausgestaltet sind als Deutsche. So wird im Bosnischen häufig zwischen den pronominalen Anredeformen $d u$ und Sie gewechselt, wohingegen im Deutschen fast ausschließlich das Distanzpronomen Sie verwendet wird.

Simona Pekarek Doehler (Neuchâtel) präsentierte in ihrem Vortrag „Documenting change over time in interactional practices: challenges for longitudinal CA", wie Veränderungen von Praktiken innerhalb sozialer Interaktionen durch longitudinale Studien erforscht werden können. An verschiedenen Beispielen veranschaulichte sie einerseits, wie kollektionenbasierte longitudinale Studien aussehen können und andererseits, welche Herausforderungen sich bei der kollektionenbasierten Korpuserstellung ergeben, wenn die sequenzielle Organisation der Interaktion sich zu jedem der aufgezeichneten Zeitpunkte unterscheidet und das Ordnungsprinzip der Analyse nicht mehr länger die sequenzielle Ordnung alleine ist, sondern die chronologische Historie.

\section{Datensitzungen}

Der Nachmittag des zweiten Tagungstages wurde durch drei parallel stattfindende Datensitzungen ergänzt, in denen Fallbeispiele aus aktuellen Forschungsprojekten unter bestimmten Forschungsaspekten gemeinsam analysiert und diskutiert wurden. Dabei ging es bei Axel Schmidt und Jörg Zinken um „Freies Spiel unter fünfjährigen und neunjährigen Kindern“, bei Ina Kordts um „Neu zugewanderte Schülerinnen in unterschiedlichen Schulformen“ und bei Helga Kotthoff und Falko Röhrs um „Vergleichende Analysen schulischer Elterngespräche“.

\section{Vorträge - Tag 3}

Der dritte Tag der Tagung wurde durch den Vortrag „Orchester- vs. Theaterproben: Vergleichende Überlegungen zu Instruktionen in Probenprozessen“ eingeleitet. Monika Messner und Anna Wessel (Innsbruck/Halle) verglichen Instruktionen der künstlerischen 
Leitungen von Schauspielensembles und Orchestern (Dirigent/innen vs. Regisseur/innen). Sie behandelten insbesondere Gemeinsamkeiten und Unterschiede von Instruktionen während des gemeinsamen Spiels. Als Instruktionen wurden Äußerungen definiert, die die Performanz der Musiker/innen und Schauspieler/innen sowie Gedanken und Vorstellungen zum Stück betreffen, die durch die künstlerische Leitung zur gemeinsamen Umsetzung genannt werden. Sie zeigten, dass Instruktionssequenzen in der asymmetrischen Interaktionssituation, in der die künstlerische Leitung stets das Vorrecht zum Sprechen hat, strukturell sehr ähnlich sind.

Ina Pick stellte in ihrem Vortrag ,Vergleichende Bestimmung des Handlungstyps Beraten: Theoretische Ergebnisse und methodische Implikationen" vor, wie sich beratende Handlungen empirisch vergleichen lassen. Den Handlungstyp Beraten typologisierte sie auf der Basis einer Merkmalsmatrix, die auf Re-Analysen bereits bestehender empirischer Untersuchungen zu beratenden Handlungen basiert. Die Typologie ermöglicht es, verschiedene Beratungstypen zu bestimmen, beratende von nicht-beratenden Handlungen abzugrenzen und die Ermittlung der Ausprägung des Beratens im jeweiligen Kontext zu bestimmen, die in verschiedenen Gesprächstypen unterschiedlich stark ausgeprägt sein kann. Überdies erläuterte sie den Nutzen visualisierend-quantifizierender Verfahren für zuvor erarbeitete empirisch-qualitative Ergebnisse.

Der Vortrag „Linguistische Analyse von Schmerzschilderungen bei Kindern und Jugendlichen“ (LASS-Studie) von Birte Schaller, Heike Knerich, Barbara Job, Joachim Opp (Bielefeld/Oberhausen) führte vor, wie durch den Vergleich linguistischer Merkmale bei Schmerzbeschreibungen von Kindern und Jugendlichen, die unter Bauchschmerzen leiden, Tendenzen, ob sie an organischen oder psychosomatischen Beschwerden leiden, beschrieben und dadurch in der Folge schneller durch die/den behandelnde Ärztin/behandelnden Arzt diagnostiziert werden können. Im Projekt LASS wurden die Arzt-PatientenGespräche, die medizinische Diagnose der Ärztin/des Arztes sowie gemalte Bilder der Kinder und Jugendlichen, die den Schmerz malen sollten, analysiert und ausgewertet. Die Analysen zeigten, dass psychosomatisch erkrankte Kinder und Jugendliche zu Beginn eines Gesprächs besonders die Schmerzintensität, Umstände und den zeitlichen Verlauf des Schmerzes relevant setzen. Demgegenüber fokussierten organisch erkrankte Kinder und Jugendliche besonders die Schmerzqualität.

Im Hinblick auf kulturspezifische Ausprägungen kommunikativer Praktiken fragte Susanne Günthner (Münster) in ihrem Vortrag ,Facetten einer kulturvergleichenden Interaktionsforschung: Chinesische und deutsche Strategien bei der kommunikativen Konstruktion sozialer Aktivitäten" danach, wie in deutschen und chinesischen Kurznachrichteninteraktionen (SMS-, WhatsApp- und WeChat-Nachrichten) kommunikative Praktiken wie nominale Selbstreferenzen, die zunächst sprachsystematische Ähnlichkeiten aufweisen können, sozio-kulturell unterschiedlich determiniert sein können. Während im Chinesischen Verwandtschaftsnamen als nominale Selbstreferenzen (auch bei nicht verwandten Personen) und performative Praktiken zur Selbst- und Fremdpositionierung eingesetzt werden, werden sie in den deutschen Daten von Sprecher/innen als ritualisierte Praktiken beispielsweise zwischen Eltern und Kindern oder unter Paaren als Liebesgeplänkel eingesetzt. Aus sprach- und kulturkontrastiver Perspektive weisen sie nicht dieselbe Performativität wie im Chinesischen auf, denn das Chinesische weist als eine 0-Anaphern-Sprache keine deiktischen Pronomina auf, sodass die pronominale Anredeform im Gegensatz zum Deutschen nicht auf soziale Beziehungs-/Hierarchierelationen hinweist. 
Abgeschlossen wurde die Tagung von Jörg Zinken und Silke Reineke, die basierend auf den Tagungsergebnissen resümierten, dass sich innerhalb der Gesprächsforschung eine Verschiebung der ausschließlich auf die Konversationsanalyse gestützten Methodologie zeigt. Die Konversationsanalyse stellte zwar in allen vorgestellten Untersuchungen die grundlegende Methode für die Analyse sprachlicher Interaktionen dar, insbesondere in Hinblick auf sprach- und kulturvergleichende Untersuchungen könne es jedoch überlegenswert sein, sie mit weiteren Methoden zu assoziieren, die es im Hinblick auf vergleichende Untersuchungen ermöglichen, den Eingang sozio-kulturellen Wissens in die Interaktion miteinzubeziehen.

Die diesjährige Arbeitstagung diskutierte somit vergleichende Untersuchungen verschiedener Vergleichsparameter wie Individuen und Gruppen, Sprachen und Kulturen, Institutionen, Praktiken sowie Entwicklungspunkte in der Konversation. Die anregenden und interessanten Vorträge zeigten, dass es fruchtbar sein kann, innerhalb der Gesprächsforschung und insbesondere in Hinblick auf sprach- und kulturvergleichende Untersuchungen, die Vorgehensweise der Konversationsanalyse mit weiteren Methoden zu verbinden. 